\title{
When cells stash their trash
}

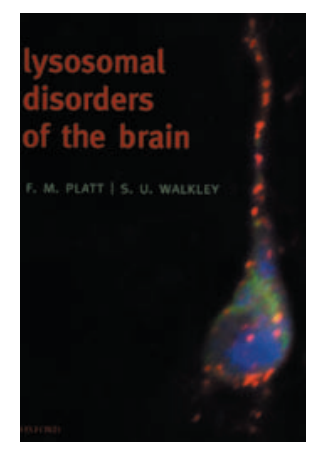

\section{Lysosomal Disorders of the Brain}

Edited by Frances Platt and Steven Walkley

Oxford University Press • 2004

$\$ 179.50 / £ 85$

\section{Anthony H. Futerman and} Gerrit van Meer

Lysosomal storage disorders (LSDs) — a class of more than 40 inherited metabolic diseases with severe pathologies, including brain dysfunction - were first described as clinical entities over a hundred years ago. Thus, it may seem strange that the dawn of a new century should see renewed interest in LSDs, marked by a notable increase in the publication of primary research articles, reviews, and now a book entitled Lysosomal disorders of the brain that is devoted to the effect of these diseases on brain function. Indeed, although the clinical course of LSDs has been extensively described over the past decades, much less is understood about the mechanisms leading from a molecular defect in lysosomal proteins to cell dysfunction and pathophysiology, especially in the brain. Thus, a recurring theme in the book is that the clinical spectrum of how these diseases affect the brain is highly variable, and it is therefore not particularly useful either as a taxonomic guide, that is, as a basis of disease classification, or as a means to understand disease mechanisms.

With this in mind, and with the historical burden of the conventional classification scheme of these diseases in view, the editors, Frances Platt and Steven Walkley, set out to discuss LSDs within the context of the molecular and cellular defects that may (or may not) be common to more than one LSD, rather than discussing each individual LSD as a biochemical and clinical entity. Thus, aficionados of Gaucher disease, the most common LSD, may be initially disappointed that their search for a chapter on 'Recent Advances on Gaucher Disease', is fruitless. However, a closer look will reveal that this disease, often considered a prototype of other LSDs, is discussed thoroughly throughout the book, in chapters that provide an overview (section I), discuss the molecular mechanisms of storage (section II), discuss pathophysiology (section III) and discuss treatment (section IV). For the most part, this ambitious approach works extremely well and the editors are hardly to be faulted that little mechanistic information is available for some of the diseases. This is

Anthony Futerman is in the Department of Biological Chemistry, Weizmann Institute of Science, Rehovot 76-100, Israel (e-mail: tony. futerman@weizmann.ac.il) and Gerritvan Meer is in the Department of Membrane Enzymology, Institute of Biomembranes, Utrecht University, Padualaan 8, 3584 CH Utrecht, The Netherlands

(e-mail:g.vanmeer@chem.uu.nl) typified by the neuronal ceroid lipofuscinoses (NCLs), in which the $c$ subunit of the mitochondrial ATP synthase accumulates, even though the defective genes in the eight or nine sub-types of this disease seem to have little to do with mitochondrial metabolism.

Another stated goal of this book is to alert cell biologists and neurobiologists to the unique insights into normal cell physiology that might be gained from studying LSDs. For instance, the book convincingly describes how perturbing lysosomal storage actually causes defects in various compartments of the endo-lysosomal system. It might be expected that delineating changes that occur in LSDs to this complex membrane system - with its vesicular connections and sorting mechanisms for proteins and lipids - would yield important information about how this system is regulated in normal cells. Lysosomal storage can result from widely different molecular defects, and over the years, the study of the molecular basis of these defects has provided many new insights into how cells run their recycling systems and dispose of their biochemical trash. In the section entitled Molecular mechanisms of storage, various chapters describe the elucidation of the catalytic activities and substrate specificities of the various lysosomal enzymes. Other chapters provide rich and unexpected insights into the chemical modifications of the enzymes and protein cofactors that are required for catalytic activity, and for transport of the enzymes to lysosomes. Finally, in a number of cases the function of the mutated protein in a particular LSD remains to be identified, which illustrates our lack of insight into the function of many classes of, notably, membrane proteins of the lysosomes.

Another important goal of the book is to draw attention to the basic cellular and biochemical mechanisms that need to be understood before effective therapies are achieved. Thus, enzyme replacement therapy, currently being used clinically or in trials for a number of LSDs, teaches us about mechanisms of receptor-mediated endocytosis; cell-mediated therapy teaches us about the challenges of understanding the complexities of the blood-brain barrier; gene therapy of LSDs has wide implications for other genetic diseases; and finally, the most recent treatment, inhibiting synthesis of stored molecules ('substrate reduction therapy') should illuminate the functions of these molecules in normal cell physiology.

In summary, this book is highly recommended, and could be read either by itself, or in conjunction with The Metabolic and Molecular Basis of Inherited Diseases, which gives a more extensive clinical and historical description of each individual LSD. As with any book in these days of internet-based searches and rapid electronic publishing, this book is not entirely up-to-date (in this case, most chapters cover the literature up to 2002), and it also lacks optimal search functions as compared to electronic formats. However, for those readers wanting to get a clear and straightforward overview of LSDs, with the added bonus of the novel classification scheme discussed above, then this well-written book is a worthwhile investment. 